\title{
A Biomechanical Analysis of Handcycling: A Case Study
}

\section{Arnaud Faupin, Philippe Gorce, Eric Watelain, Christophe Meyer, and Andre Thevenon}

\begin{abstract}
The aim of this study was to investigate muscle activity, kinematic, and handgrip-force pattern generation during handcycling. One able-bodied participant performed a 1-min exercise test on a handcycle at 70 revolutions per minute. This article proposes an original data collection and analysis methodology that gathers synchronized kinematics, kinetics, and electromyography. Such data, which most often appear complex, are easily summarized using this methodology. This preliminary study has an new setup and offers good indications on the biomechanical pattern for handcycling movement analysis.
\end{abstract}

Keywords: handcycling, kinematics, kinetics, EMG

It has been demonstrated that manual wheelchair propulsion has a low mechanical efficiency (van der Woude et al., 2001). Thus, research has shown renewed interest in complementary propulsion strategies, such as the application of an arm crank system used in handcycling, in an effort to increase the efficiency of upper extremity ambulation. Handcycling has enhanced mobility in daily life activities and increased training and sports opportunities for wheelchair-dependent users. However, research on handcycling is scarce and limited to physiological studies evaluating the metabolic responses and race performance of handcycle athletes (Janssen et al., 2001), the influence of different cadence strategies in handcycling (Verellen et al., 2004a), comparing gross mechanical

Faupin is with Laboratoire d'Etudes de la Motricité Humaine (LEMH), Equipe d'Accueil 3608, "Activité Physique, Sport, Santé", Faculté des Sciences du Sport et de l'Education Physique, Université de Lille 2, Ronchin, France, and Laboratoire d'Automatique de Mécanique et d'informatique Industrielles et Humaines (LAMIH-UMR CNRS 8530), Université de Valenciennes, Le Mont Houy, Valenciennes, France. Gorce is with Laboratoire de biomodelisation et ingénierie des handicaps « handibio », Equipe d'Accueil 4322, Université Toulon et du Var, La Garde, France. Watelain is with Laboratoire de biomodelisation et ingénierie des handicaps « handibio », Equipe d'Accueil 4322, Université Toulon et du Var, La Garde, France, and Laboratoire d'Automatique de Mécanique et d'informatique Industrielles et Humaines (LAMIH-UMR CNRS 8530), Université de Valenciennes, Le Mont Houy, Valenciennes, France. Meyer is with Department of Rehabilitation Sciences, Faculty of Physical Education and Physiotherapy, Katholieke Universiteit Leuven, Leuven, Belgium. Thevenon is with Laboratoire d'Etudes de la Motricité Humaine (LEMH), Equipe d'Accueil 3608, "Activité Physique, Sport, Santé", Faculté des Sciences du Sport et de l'Education Physique, Université de Lille 2, Ronchin, France, and Centre Hospitalier Régional Universitaire de Lille Service Médecine Physique et Réadaptation (SMPR-CHRU), Unité d'analyse du mouvement, Lille, France. efficiency during handcycling compared with manual wheelchair propulsion (Mukherjee \& Samanta, 2001; Dallmeijer et al., 2004), and comparing asynchronous and synchronous cranking during handcycling (van der Woude et al., 2000; Abel et al., 2003).

Thus far, few studies about the biomechanics of handcycling exist in the literature (van der Woude et al., 2001, 2006). At present, very few data concerning the kinematic (Faupin et al., 2004, 2006, 2008), kinetic (Verellen et al., 2004b), and surface electromyographic (EMG) (DeCoster et al., 1999) parameters of handcycling are available.

However, to the best of our knowledge, no one has synchronized the muscle activity patterns, and kinematic and force-generation patterns during handcycling. Understanding the biomechanics of handcycling propulsion is important to improve the quality of life for wheelchair users in general, and to optimize performance and especially in the ergonomic optimization of the position of the user on his or her handcycle. This exploratory case study, which focuses on a biomechanical analysis of handcycling at a low level of propulsion, first aims to propose an original methodology that combines kinetics, kinematics, and electromyography acquisition and presentation. Second, the initial results of this pilot study should provide information about specific patterns in one able-bodied subject's handcycling and offer some shortand long-term perspectives on investigations for specific users, such as spinal cord-injured athletes.

\section{Methodology}

\section{Experimental Protocol}

One able-bodied participant (age: 24 years, mass: $65 \mathrm{~kg}$, height: $170 \mathrm{~cm}$ ), inexperienced in handcycling, was fully informed of any risks before giving her written informed consent to participate in this experiment. Once settled onto the handcycle, which was connected to a home trainer, the participant had 10 min to become accustomed 
to the equipment. This participant then performed a 1-min exercise test at a crank rate of $70 \mathrm{rpm}$, imposed by metronome. The experimental procedures were approved by the local ethics committee and complied with the ethical standards of the 1975 Helsinki Declaration modified in 1983.

\section{Instrumentation}

An adjustable sport handcycle with synchronous crank montage was used in this study (Sopur, Spirit 470, Sunrise Medical of Heidelburg, Germany). The backrest of the handcycle was tilted backward at an angle of $45^{\circ}$. The handcycle was connected to a computer-linked ergocycle (Elite, Axiom, Italy). The Elite Axiom ergocycle (Bertucci et al., 2005) was equipped with a motor unit that imposed a constant rolling resistance on the front wheel. The rolling resistance was also chosen by the researcher ( $1 \%$ in the current study).

Kinematic, kinetic, and EMG data were collected on the right side during the second $30 \mathrm{~s}$ of the 1-min exercise test. Five complete crank cycles were analyzed for all parameters. MATLAB (MathWorks, Inc., Natick, MA) programs were used for the data calculations.

\section{Kinematic Data}

The 3-D movement analysis was performed using a Vicon 370 system (Oxford Metrics, Oxford, UK) that comprised six digital cameras. Figure 1 shows the positioning of the 22 anatomical and technical markers used. Two reflective markers attached to the handgrip and one on the crank axis allowed the handgrip's angular position (Figure 1) to be obtained and the handgrip orientation $\left(\theta_{2}\right)$ and the crank angle $\left(\theta_{1}\right)$ to be measured.

Anatomic frames were defined according to the International Society of Biomechanics recommendations (Wu \& Cavanagh, 1995). The upper part of the human body was considered to be an articulated system composed of rigid bodies corresponding to the following body segments: head, trunk, arm, forearm, and hand. Thus, Euler angles were chosen to describe the relative movement of the body segments, and the global optimization method was used to minimize measurement errors due to sliding skin (Roux et al., 2002). During the test, the maximum and minimum angles and the total range of motion in degrees were calculated for the shoulder (flexion/extension, internal/external rotation, and abduction/adduction), the elbow (flexion/extension) and the wrist (flexion/extension, radial/ulnar deviation). Kinematic data were filtered using a fourth-order digital Butterworth filter with a cutoff frequency of $6 \mathrm{~Hz}$ (Cooper et al., 2002). Kinematic data (Figure 2) were averaged for five consecutive cycles and normalized according to the crank angle $\left(0-360^{\circ}\right)$.

\section{Kinetic Data}

A freely rotating instrumented right dynamometric handgrip (Sensy, 9PED version [aluminum], France) with an attached handgrip was used to measure normal and tangential forces. The handgrip was calibrated by hanging weights (from 0 to $1500 \mathrm{~N}$ ) to a dynamometric calibration device fastened to the handgrip, and voltage outputs from foil strain gages were amplified and then recorded. A linear regression equation showed that handgrip voltage was a strong predictor of handgrip force $\left(R^{2}\right.$ $=.99)$. The dynamometric handgrip was connected to the Vicon system for synchronous acquisition of all the different types of data. Kinematic data were collected at a frequency of $60 \mathrm{~Hz}$, whereas forces and EMG data were collected at a frequency of $1200 \mathrm{~Hz}$. These 1200$\mathrm{Hz}$ signals were then low sampled (from 1200 to $60 \mathrm{~Hz}$ ), using a cubic spline function for synchronization with the kinematic data. Kinetic data were filtered through a fourth-order digital Butterworth filter with a cutoff frequency of $10 \mathrm{~Hz}$ (Cooper et al., 1998).

The handgrip reference system measured normal $\left(F_{n}\right)$ and tangential $\left(F_{t}\right)$ forces. These measured force components, along with the handgrip orientations $\left(\theta_{2}\right)$ and the crank angle $\left(\theta_{1}\right)$, were used to calculate the total $\left(F_{t o t}\right)$, radial $\left(F_{r a d}\right)$ and the effective $\left(F_{e f f}\right)$ force for the global reference system (GRS). The term $F_{t o t}$, which is the total force applied to the handgrip, was calculated mathematically using the vector sum of the force components (in newtons):

$$
F_{\text {tot }}=\sqrt{\left(F_{\text {eff }}^{2}+F_{\text {rad }}^{2}\right)}
$$

According to the literature on manual wheelchair propulsion or cycling (Boninger et al., 1997; Zameziati

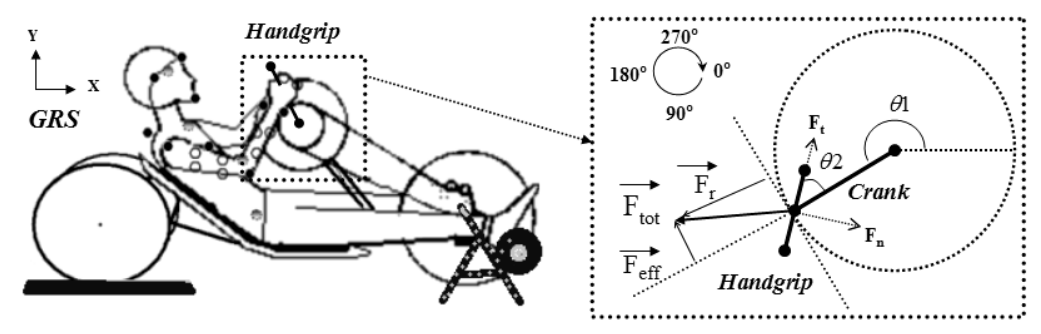

Figure 1 - Schematic setup. Kinematic marker ( $\bullet$ anatomical, o technical) positions are indicated on the left side and handgrip kinetics, on the right side. The total force $\left(F_{t o t}\right)$, the radial force $\left(F_{r}\right)$, and the effective force $\left(F_{\text {eff }}\right)$ were calculated from the normal force $\left(F_{n}\right)$, the tangential force $\left(F_{t}\right)$, the handgrip orientation $\left(\theta_{2}\right)$ and the crank angle $\left(\theta_{1}\right)$ in the global reference system $($ GRS). $x$ and $y$ are three-dimensional coordinates in the GRS. 

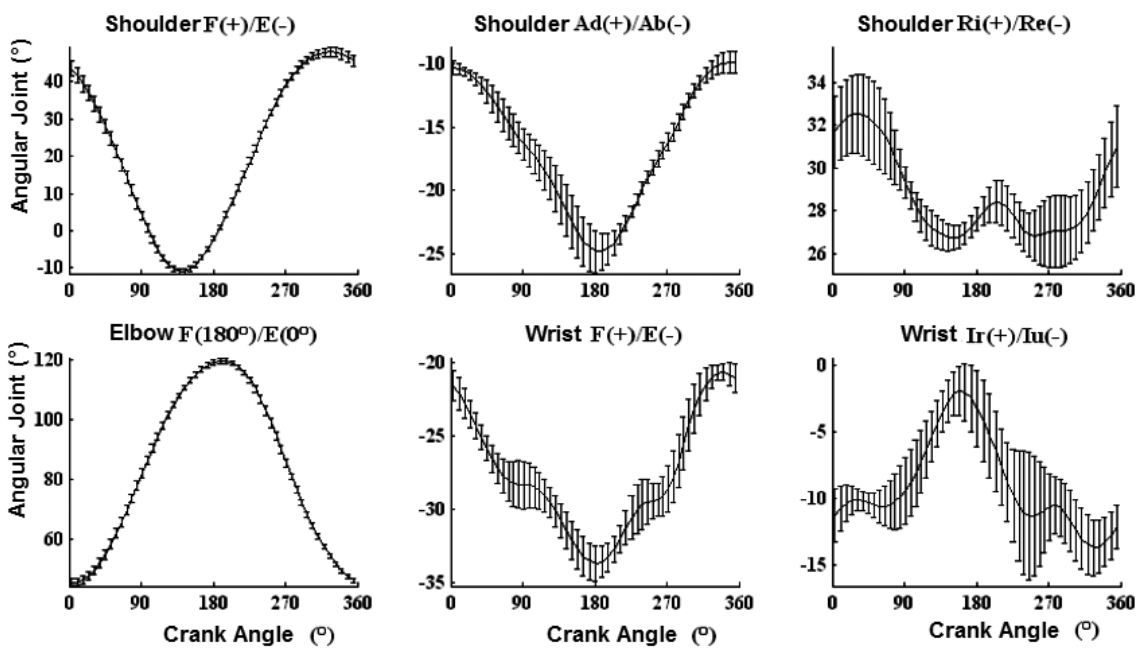

Figure 2 - Crank angle $\left(0-360^{\circ}\right)$ versus angular variability (mean and standard deviation for five consecutive cycles) for the shoulder, elbow, and wrist. F/E: flexion / extension; Ad/Ab: adduction / abduction; Ri/Re: internal rotation / external rotation; Ir/ $\mathrm{Iu}$ : radial inclination / ulnar inclination; $(+)$ and $(-)$ are associated with an angular joint corresponding to, for example, $\mathrm{F}(+) / \mathrm{E}(-)$ : flexion (with maximum or minimum positive values) / extension (with maximum or minimum negative values).

et al., 2006), the ratio between the total force and the force tangential to the crank rotation-or the effective force $F_{\text {eff }}$ - the only force component that contributes to the forward motion of the handcycle is used to calculate the movement effectiveness. Therefore, the following equation was used to calculate the 2-D fraction effective force $\left(F E F_{2 D}\right)$ during the complete cycle:

$$
F E F_{2 D}=\frac{\int_{0}^{360} F_{e f f}\left(\theta_{1}\right) \cdot d \theta_{1}}{\int_{0}^{360} F_{t o t}\left(\theta_{1}\right) \cdot d \theta_{1}} \times 100
$$

\section{EMG Data}

Muscular activity was recorded with the help of a surface EMG model MA300 (Motion Laboratory Systems, Inc.) using pre-gelled disposable surface electrodes. Electrodes were positioned with an interelectrode distance of 20 $\mathrm{mm}$ on the following muscles: biceps brachii (Bi), triceps brachii (Ti), pectoralis major (Pm), upper trapezius (Tr), anterior deltoid (Da), and posterior deltoid (Dp). The linear envelope of the raw signal was obtained by full-wave rectifying signals. Electromyographic (Figure 3) data were averaged for five consecutive cycles and normalized according to the crank angle $\left(0-360^{\circ}\right)$ for duration and maximal voluntary contraction for range. Electromyographic activation was defined as an activity with an intensity equivalent to at least $5 \%$ of the muscle test level for duration of minimum $5 \%$ of the entire cycle (Mulroy et al., 1996).

\section{Statistical Analysis}

The average and standard deviations over the five consecutive complete crank cycles were calculated. To evaluate the consistency of the value within-cycle force pattern distribution, the variation coefficient (VC) was calculated as a ratio of the mean standard deviation to the average; this is a dispersion indicator (Verellen et al., 2004b).

\section{Results}

Figure 4 summarizes the relationships between the forces applied on the cranks, the activated muscles, and the angular parameters of the upper body, thus improving the understanding of the movement pattern. The different activated muscles are presented inside a representation of the crank trajectory. Solid lines indicate the direction and magnitude of the resultant force on the handgrip. Linked to the direction of the resultant force, the $F E F_{2 D}$ is equal to $85 \%$, with a $\mathrm{VC}$ equal to $11 \%$. The four diamonds, each pair connected by a solid black line, indicate the crank orientation and position.

\section{Discussion}

It should be emphasized that the data presented here are from a single able-bodied participant. Therefore we do not know if they are completely transferable to wheelchair-dependent users with trunk and/or upperlimb disabilities. Nevertheless, resulting from this development, handcycling as a sporting discipline has spread to a larger population. Hence, handcycling competitions are not only for people with limited trunk function (such as those with spinal cord injuries), but are also open to disabled athletes with good trunk control (such as poliomyelitis sufferers or leg amputees). Furthermore, outside International Paralympic Committee events, the European Handcycle Circuit includes able-bodied athletes in its championships-this further boosts the 

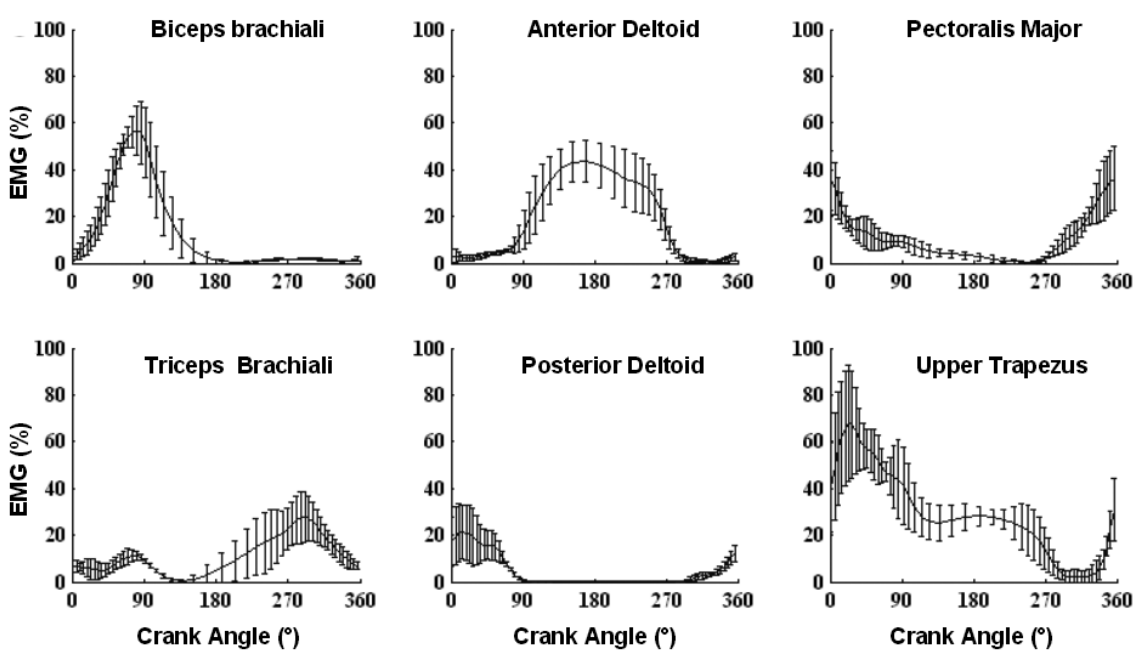

Figure 3 - The EMG pattern normalized by maximal voluntary contraction for the six muscles recorded, in terms of the crank angle $\left(0-360^{\circ}\right)$.

sport's development and increases interest in handcycling for the nondisabled.

In this handcycling study, the calculated $F E F_{2 D}$ value $(85 \%)$ is higher than the values existing in manual wheelchair propulsion ranging from $47 \%$ (Wu et al., 1998) to $81 \%$ (Veeger et al., 1992). It consequently seems that, regardless of a participant's experience, force application is highly efficient in handcycling. Values in handcycling are more comparable to those of cycling (Coyle et al., 1991), as differentiated from those of manual wheelchair propulsion, which might be explained by a closed loop movement. Also comparable to cycling (Zameziati et al., 2006) is that force application appears continuous throughout the propulsion cycle (Figure 4).

In addition, using the variation coefficient-which is considered as a key parameter in assessing the efficiency of cyclic movement such as cycling (Caldwell et al., 1998) or handcycling (Verellen et al., 2004b; Bafghi et al., 2008)—we considered the reproducibility of the force pattern as consistent given $\mathrm{VC}$ is equal to $11 \%$ for $F E F_{2 D}$. Such finding is in line with the literature (Verellen et al., 2004b).

Figure 4 shows muscle activation during five complete cycles. One can see that the handgrip orientation can be broken down into two phases: the first between $0^{\circ}$ and $180^{\circ}$ and the second between $180^{\circ}$ and $360^{\circ}$. The handgrip orientation is related with the wrist kinematics (flexion/extension, radial/ulnar deviation); these two transitional phases coincide with the full flexion and extension of the elbow (Figure 2). Muscle $\mathrm{Bi}$ is activated between $0^{\circ}$ and $180^{\circ}$, whereas logically $\mathrm{Ti}$ is activated between $180^{\circ}$ and $360^{\circ}$. Using only these parameters, we could define a pull phase corresponding to the activity of Bi between $0^{\circ}$ and $180^{\circ}$ and a push phase corresponding to the activity of $\mathrm{Ti}$ between $180^{\circ}$ and $360^{\circ}$. In this case, $0^{\circ}$ (i.e., $360^{\circ}$ ) and $180^{\circ}$ would correspond to two transition phases. We found weak concomitant Ti activity with $\mathrm{Bi}$ activity, which indicates a co-contraction time lapse (Figure 4). This phenomenon can be explained by Bernstein's theory, which it says that a novice participant reduces the number of degrees of freedom in a new task by muscle co-contractions. However, handcycling is a guided movement with a limited degree of freedom, and we did not observe co-contraction for the other antagonist pairs. This observation must, of course, be confirmed with a larger and more experienced population.

Consequently, this study offers some short- and longterm perspectives. A more substantial experimental database would allow us to develop a specific biomechanical model. Parameters related to handbike configuration, such as crank adjustments (height, width, shape, with handles, etc.), the backrest angle, or the distance of the seat compared with the cranks will be taken into consideration in modeling to highlight their respective influences. Parameters related to a participant's morphology (center of gravity of the trunk, of each limb segment) and to motor and functional user's abilities will also require particular attention. Moreover, dynamic movement modeling is nowadays a subject of research, the results of which have effects on the ergonomic optimization of several mechanisms. This kind of work could also have industrial repercussions on vehicle design for motor-disabled users. Therefore, we invite regular handbikers to be mindful of these considerations, be it during a reeducation program or during sports training.

To conclude, this preliminary study has an original setup and offers good indications on the biomechanical pattern for handcycling movement, which has not been extensively described in the literature. The methods used, sometimes appearing complex, are innovative and combine kinetics, kinematics, and electromyography. Their results are summarized in an easy way. However, future experiments need to be performed on several novice subjects and on persons from specific disease groups, particularly with amputees and persons with spinal cord injuries, the principal groups who use handbikes. 

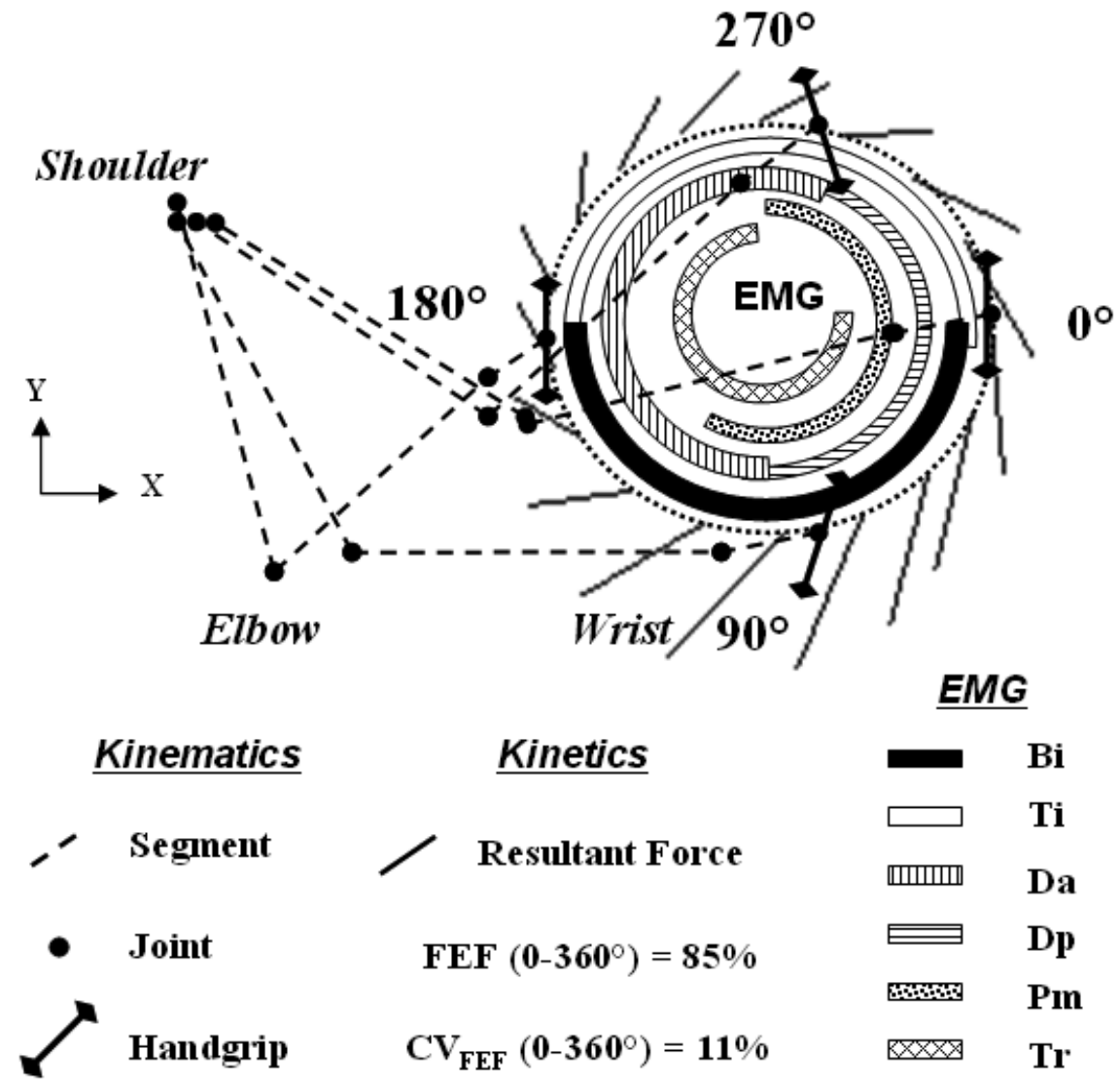

Figure 4-Muscular activity, segmental displacements, and force applications over five consecutive cycles projected in the sagittal plane for the able-bodied subject. $\mathrm{cv}_{\text {fef }}$ is the variability coefficient of 2-d Fraction Effective Force $\left(F E F_{2 D}\right)$. Bi: biceps brachii; Ti: triceps brachii; Pm: pectoralis major; Tr: upper trapezius; Da: anterior deltoid; Dp: posterior deltoid. $x$ and $y$ are three-dimensional coordinates in the global reference system.

\section{Acknowledgments}

The authors thank Olivier Remy-Neris and André Thevenon for their scientific help and Stéphane Bouilland for technical assistance during the experiment. This project was performed in the J. Calvé Center (Groupe Hopale, 72 esplanade Parmentier, 62608 Berck sur mer). The authors also thank the Institut Garches, which allowed us to perform this project through its financial participation (Hôpital Raymond Poincaré, 104, boulevard Raymond-Poincaré, 92380 Garches.)

\section{References}

Abel, T., Vega, S.R., Bleicher, I., \& Platen, P. (2003). Handbiking: physiological responses to synchronous and asynchronous crank montage. European Journal of Sport Science, 3, 1-9.

Bafghi, H.A., de Haan, A., Horstman, A., \& van der Woude, L. (2008). Biophysical aspects of submaximal hand cycling. International Journal of Sports Medicine; Epub ahead of print.

Bertucci, W., Duc, S., Villerius, V., \& Grappe, F. (2005). Validity and reliability of the Axiom PowerTrain cycle ergometer when compared with an SRM powermeter. International Journal of Sports Medicine, 26, 59-65.
Boninger, M.L., Cooper, R.A., Robertson, R.N., \& Rudy, T.E. (1997). Wrist biomechanics during two speeds of wheelchair propulsion: an analysis using a local coordinate system. Archives of Physical Medicine and Rehabilitation, 78, 364-372.

Caldwell, G.E., Li, L., McCole, S.D., \& Hagberg, J.M. (1998). Pedal and crank kinetics in uphill cycling. Journal of Applied Biomechanics, 14, 245-259.

Cooper, R.A., DiGiovine, C.P., Boninger, M.L., Shimada, S.D., \& Robertson, R.N. (1998). Frequency analysis of 3-dimensional pushrim forces and moments for manual wheelchair propulsion. Automedica, 16, 355-365.

Cooper, R.A., DiGiovine, C.P., Boninger, M.L., Shimada, S.D., Koontz, A.M., \& Baldwin, M.A. (2002). Filter frequency selection for manual wheelchair biomechanics. Journal of Rehabilitation Research and Development, 39, 323-336.

Coyle, E.F., Feltner, M.E., Kautz, S.A., Hamilton, M.T., Montain, S.J., Baylor, A.M., et al. (1991). Physiological and biomechanical factors associated with elite endurance cycling performance. Medicine and Science in Sports and Exercise, 23, 93-107.

Dallmeijer, A.J., Zentgraaff, I.D., Zijp, N.I., \& Van der Woude, L.H. (2004). Submaximal physical strain and peak performance in handcycling versus handrim wheelchair propulsion. Spinal Cord, 42, 91-98. 
DeCoster, A., Van Laere, M., \& Blonde, W. (1999). Electromyographic activity of shoulder girdle muscles during handbiking. In van der Woude, L.H.V., Hopman, M.T.E., \& van Kemenade, C.H., Biomedical aspects of manual wheelchair propulsion: The state of the art II (pp. 138142). Amsterdam, Washington, DC: IOS Press.

Faupin, A., Campillo, P., Thevenon, A., Gorce, P., Bouilland, S., \& Rémy-Néris, O. (2004). The effects of backrest adjustments on kinematic parameters produced during the handcycle sprinting. Journal of Rehabilitation Research and Development, 41(Suppl. 2), 22. (Third International Congress Restoration of [Wheeled] Mobility in SCI Rehabilitation: State of the Art III, Vrije Universiteit, Amsterdam, the Netherlands, April 19-21, 2004.)

Faupin, A., Gorce, P., Campillo, P., Thevenon, A., \& RemyNeris, O. (2006). Kinematics analysis of handbike propulsion in various gear ratio: implication to joint pain. Clinical Biomechanics (Bristol, Avon), 21, 560-566.

Faupin, A., Gorce, P., Meyer, C., \& Thévenon, A. (2008). Effect of backrest positioning and gear ratio on non-disabled subjects' handcycling sprinting performance and kinemtics. Journal of Rehabilitation Research and Development, 45, 109-116.

Janssen, T.W., Dallmeijer, A.J., \& van der Woude, L.H. (2001). Physical capacity and race performance of handcycle users. Journal of Rehabilitation Research and Development, 38, 33-40.

Mukherjee, G., \& Samanta, A. (2001). Physiological response to the ambulatory performance of handrim and arm-crank propulsion systems. Journal of Rehabilitation Research and Development, 38, 391-399.

Mulroy, S.J., Gronley, J.K., Newsam, C.J., \& Perry, J. (1996). Electromyographic activity of shoulder muscles during wheelchair propulsion by paraplegic persons. Archives of Physical Medicine and Rehabilitation, 77(2):187-193.

Roux, E., Bouilland, S., Godillon-Maquinghen, A.-P., \& Bouttens, D. (2002). Evaluation of the global optimisation method within the upper limb kinematics analysis. Journal of Biomechanics, 35(9), 1279-1283. doi:10.1016/S00219290(02)00088-X

Van der Woude, L.H.V., Bosmans, I., Bervoets, B., \& Veeger, H.E.J. (2000). Handcycling: Different modes and gear ratios. Journal of Medical Engineering \& Technology, 24, 242-249.

Van der Woude, L.H., Veeger, H.E., Dallmeijer, A.J., Janssen, T.W., \& Rozendaal, L.A. (2001). Biomechanics and physiology in active manual wheelchair propulsion. Medical Engineering \& Physics, 23, 713-733.

Van der Woude, L.H., de Groot, S., \& Janssen, T.W. (2006). Manual wheelchairs: Research and innovation in rehabilitation, sports, daily life and health. Medical Engineering \& Physics, 28, 905-915.

Verellen, J., Theisen, D., \& Vanlandewijck, Y. (2004a). Influence of crank rate in hand cycling. Medicine and Science in Sports and Exercise, 36, 1826-1831.

Veeger, H.E., van der Woude, L.H., \& Rozendal, R.H. (1992). A computerized wheelchair ergometer. Results of a comparison study. Scandinavian Journal of Rehabilitation Medicine, 24, 17-23.

Verellen, J., Gers, B., Van de Vliet, P., \& Vanlandewijck, Y. (2004b). Consistency of the within cycle torque distribution pattern during hand cycling: a pilot study. European Bulletin in Adapted Physical Activity, 3, 5p www.bulletin-apa.

Wu, G., \& Cavanagh, P.R. (1995). ISB recommendations for standardization in the reporting of kinematic data. Journal of Biomechanics, 28, 1257-1261.

Wu, H.W., Berglund, L.J., Su, F.C., Yu, B., Westreich, A., Kim, K.J., et al. (1998). An instrumented wheel for kinetic analysis of wheelchair propulsion. Journal of Biomechanical Engineering, 120, 533-535.

Zameziati, K., Mornieux, G., Rouffet, D., \& Belli, A. (2006). Relationship between the increase of effectiveness indexes and the increase of muscular efficiency with cycling power. European Journal of Applied Physiology, 96, 274-281. 